\title{
Preferences of speech and language therapists for telepractice in the COVID-19 pandemic and factors affecting their acceptance of the delivery model
}

\author{
Mehmet Emrah Cangi ${ }^{1 \oplus,}$ İbrahim Can Yaşa $^{2} \odot$, Ayşe Işıldar ${ }^{3} \odot$ \\ ${ }^{1}$ Department of Speech and Language Therapy, Üsküdar University, Faculty of Health Sciences, Istanbul, Turkey \\ ${ }^{2}$ Department of Speech and Language Therapy, Bahçeşehir University, Faculty of Health Sciences, Istanbul, Turkey \\ ${ }^{3}$ Department of Speech and Language Therapy, Medipol University, Faculty of Health Sciences, Istanbul, Turkey
}

\begin{abstract}
Objectives: With the COVID-19 pandemic, telepractice became a great option in speech-language therapy services, as in many healthcare utilities. However, the transition to this service model did not occur at a similar rate for every clinician. Therefore, this study aimed to determine the experiences, preferences and factors affecting the acceptance of speech-language therapists (SLT) regarding telepractice in Turkey.

Methods: Sixty-seven SLTs were presented with a questionnaire that addressed the professional tendencies, experiences and views on telepractice of them. Descriptive statistics regarding the preferences and experiences of SLTs were calculated. Moreover, factors that might be related to the number of sessions they held at the pandemic were examined with the Chi-squared test.

Results: The speech-language disorders that SLTs find the most suitable for telepractice were fluency disorders, voice disorders and speech sound disorders. Groups that SLTs deemed most suitable for receiving telepractice in terms of age were 12-21, 22-64 and 7-11, respectively. A significant relationship was found between the frequency of online meetings and telepractice sessions before the pandemic and the number of sessions during the pandemic. Furthermore, a significant relationship also was found between satisfaction with using clinician skills in telepractice and the number of telepractice sessions during the pandemic.

Conclusions: The importance of the first experiences of SLTs in the acceptance of the delivery method emerged. The necessity of in-service trainings and exemplary models to improve attitudes emerged. With these trainings, ensuring security, standardizing practices and increasing qualified services will be provided as well. Keywords: Telepractice, speech and language therapy, COVID-19 pandemic, technology acceptance, clinicians' perspective
\end{abstract}

A fter COVID-19 was first identified in Wuhan province of China, it spread all over the world at an unexpected speed. Thus, this process caused a great change in the daily lives of individuals in Turkey as well as in the world, and deeply affected their access to services such as education and health $[1,2]$.

One of the professional professions that provide services in the field of health is speech and language therapy (SLT). Clinicians in this field actively serve in the evaluation and therapy of communication, lan- 
guage, speech, voice and swallowing disorders in the clinical field [3]. Before the pandemic, these services were widely offered in-person in clinics, rehabilitation centers or hospitals [4]. However, SLTs work in inperson sessions in environments with high risk of transmission of the virus due to services that require close contact like many healthcare professionals. For this reason, as recommended by the American Speech and Language Association (ASHA) [5] and the Association of Speech and Language Therapists (DKTD) in Turkish [3], in-person services were decommissioned in Turkey as a precaution against the risk of those clinicians getting infected. In this context, the search for alternative services increased in order to maintain the therapy service.

An important alternative to in-person services is telepractice (i.e. telehealth, telemedicine and telerehabilitation). This service model is the usage of telecommunication technologies for evaluation and therapy between clinician and client, and for consultation between clinicians [6]. There is evidence that this model can be suitable and effective in many disorders. Voice disorders [7], dysphagia [8], stuttering [9], aphasia [10], communication, speech and language impairments due to the autism spectrum disorder [11], and speech sound disorders [12] can be shown as examples to these disorders.

Telepractice is seen as an alternative service option with many advantages [13]. In some clients, telepractice may be the main way for clients to reach services, just as in the circumstances of the COVID-19 pandemic [5]. In many studies, it is emphasized that the main advantage of telepractice is access to services and flexibility of time and place. Other advantages are reported as cost efficiency and user satisfaction [1317]. However, there are also many limitations regarding telepractice. Some examples that can be given to this situation are that the application protocols are not clear yet, problems in material selection, technological problems, uncertainty in cost-benefit analysis, unclear candidate criteria and difficulties in ensuring active participation of the client over the Internet [18-20]. Studies examining the experience of clinicians regarding telepractice services have an important place in the literature of SLT. Wales et al. [21] reviewed studies on access to SLT services through telepractice in primary school children. The authors reported that there was some promising evidence that telepractice could be used in this group. Weidner and Lowman [22] reviewed the role of some factors in telepractice services for adults. The main categories were issues such as client types, intervention approaches, and technology. The authors reported that there was evidence for the usage of telepractice in the management of many diseases such as aphasia, dysphagia and Parkinson's disease. Group therapies are also preferred in some studies. The authors also reported that teleconferencing systems such as Zoom and Adobe Connect are preferred in telepractice.

As in any new technology, determining the factors that make clinicians and clients accept this model is seen as an important issue regarding telepractice. For example, some socio-demographic characteristics such as education level, culture, age or gender were found to be effective in technology acceptance [22, 23]. As another example, Tucker [24] concluded that telepractice is mostly preferred by young SLTs. The fact that they are more competent and willing to use technology was stated as the reason for this. Another remarkable point in this context is the experience in technology according to Sun and Zhang [25] and Tucker [18]. Individuals who spend more time with these technologies have higher levels of acceptance. Technology acceptance levels were also discussed in some models. For example, Dunkley et al. [26] discussed their telepractice experiences in SLT within the scope of the Technology Acceptance Model [27]. In this model, the two most critical components in the acceptance of technology are specified as "perceived ease of usage" and "perceived usefulness" regarding the technology in question. Dunkley et al. [26] also reached conclusions supporting this model.

There are also various barriers and concerns about providing telepractice services. One of the most important concerns about telepractice is related to the level of the therapeutic relationship established with the client. However, the first evidence is that there is not a great deficiency in telepractice in this regard [28, 29]. Moreover, there were many suggestions on how to improve the relationship in telepractice. For instance, in one study, SLTs emphasized the importance of staying in touch with families and sharing phone or e-mail information [29]. Another concern is that service delivery with telepractice will have a negative im- 
pact on the therapy process and clinician skills. However, it is argued that this barrier will decrease as technology develops [30, 18].

In Turkey, there is only one work containing the mixed-study method on telepractice in the SLT field [4]. In this study, the effectiveness of stuttering therapy through telepractice in adults and the participatory and clinician perspectives on this service were examined. Consequently, evidence was obtained that telepractice could be as effective as in-person therapy in adult stuttering and that this delivery method might be suitable for stuttering therapy.

\section{Purpose}

The purpose of this study was to examine the preferences and acceptance processes of SLTs who use telepractice, such as client groups, session duration or software used during the COVID-19 process. In line with the telepractice literature, some study questions and hypotheses regarding possible relationships between these variables were defined:

1. Does the number of telepractice sessions that SLTS do after the pandemic change according to their 'demographic characteristics'?

According to the hypothesis determined regarding this study question, the number of telepractice sessions performed after the pandemic does not change according to demographic characteristics $(\mathrm{H} 0)$. In this decision, it was effective that the ratio of women to men was not balanced among the participants and that the working group was mostly composed of young participants.

2. Does the number of telepractice sessions performed by SLTs after the pandemic change according to their "online meeting and telepractice experience" and "computer use skills they perceive about themselves"?

According to the hypothesis related to the second study question, it is expected that the number of telepractice sessions performed by SLTs after the pandemic will change according to their "online meeting and telepractice experience" and "computer use skills they perceive about themselves (H1).

3. Do SLTs' satisfaction levels in telepractice-related issues (development of the client, therapeutic communication, ability to use clinician skills, software used and financial gain) vary according to the number of telepractice sessions they have held in the last month?

In the hypothesis related to the third study question, a higher level of satisfaction with this service delivery model of SLTs who perform a higher number of telepractice sessions after the pandemic is expected (H1).

In this pandemic period which significantly affected the level of SLT service, exposing the factors that affect acceptance levels for the SLT telepractice in Turkey is expected to contribute to the development and future maintenance of these services. Moreover, the groups to which the SLTs will provide with this service, the time they schedule, the approaches they prefer, their orientation to use software or hardware will provide an "initial guide" for clinicians who want to provide telepractice services.

\section{METHODS}

\section{Participants}

The participant group of the study consisted of 67 SLTs between the ages of 22-47. The average age of the participants was $29.57 \pm 6.78$ years. The basic criteria for the inclusion of SLTs in the study were determined as "to have at least a bachelor's degree in the field of SLT" and "to apply at least one session of telepractice". Participants were selected using the appropriate sampling method [31]. While 51 of the participants were women, 16 of them were men. Furthermore, 32 of this group were undergraduate, 34 had a master's degree and 1 was a doctoral graduate.

\section{Research Ethics}

The approval of the ethics committee of the study was obtained from Üsküdar University, Non-Interventional Research Evaluation Board (Number: 61351342 / 2020-244). The study was conducted in accordance with the Declaration of Helsinki.

\section{Data Collection Tools}

In order to collect the data, the questionnaire called "The Experiences of Speech and Language Therapists Regarding Telepractice Services in the COVID-19 Pandemic" was used. In the process of developing the questionnaire, the telepractice literature 
$[16,18,19,24]$ and guidelines on the maintenance of SLT services in the COVID-19 pandemic published by ASHA [5] and DKTD (ASLT) [3] were reviewed. Afterwards, related topics were discussed by the authors and various themes were determined. After the questionnaire items were written, clinician opinions were received from three experienced academicians. Finally, a pilot study was conducted with three experienced clinicians and the questionnaire was finalized in line with the feedback received.

The questionnaire consisted of four sub-dimensions which were demographic and occupational information, overview, disorders and satisfaction: (1) Within the scope of demographic information, there were multiple-choice questions and question that they could choose more than one answer about the age, gender, professional experience, and types of clients they worked with. (2) In the overview section, there were multiple choice questions that evaluate the general view of SLTs regarding telepractice. (3) In the disorders section, questions about the suitability of speech and language disorders to telepractice were included with scoring based on five-point Likert grading. (4) In the satisfaction section, there was a five-point Likert scale in which SLTs scored their satisfaction levels in various subjects from telepractice.

\section{Procedure}

The survey was uploaded to Google Surveys and the link of the survey was shared in professional email groups and social media accounts. When the targeted number of participants was reached, the questionnaire was closed to answer.

A directive was presented to the participants at the beginning of the survey. In the directive, information was given on the purpose of the questionnaire, participant criteria, and average response time and data confidentiality. No negative feedback was received from the participants that required correction.

\section{Statistical Analysis}

Descriptive statistics such as frequency, percentage and ratio were calculated in the analysis of the data. Participants' age, gender, perceived computer use skills, online meeting and telepractice experience before COVID-19, and the number of therapies and satisfaction levels of the COVID-19 pandemic were compared with the Chi-square test.

\section{RESULTS}

In this study, in which SLTs' preferences for telepractice services and their experiences regarding acceptance processes in the COVID-19 pandemic were examined, four sub-dimensions addressing the demographic and professional information of SLTs', their general view of telepractice, the disorders in which telepractice was deemed appropriate and their satisfaction level with telepractice were evaluated.

Findings on demographic and professional characteristics of the participants

Findings regarding the demographic information and professional characteristics of the participants were presented in Table 1.

\section{At what level did SLTs see their computer skills?}

SLTs often saw their computer use skills as normal or better. The most preferred option was 'good' level $(n=37)$. The number of SLTs $(n=15)$ using comput-

Table 1. Findings on demographic and professional characteristics of the participants

\begin{tabular}{lcc}
\hline Variable & $\mathbf{n = 6 7}$ & $\mathbf{\%}$ \\
\hline Gender & & \\
\hline Woman & 51 & 76.1 \\
Man & 16 & 23.9 \\
Professional experience & & \\
1-2 years & 31 & 46.3 \\
3-5 years & 14 & 20.9 \\
\hline 6-10 years & 12 & 17.9 \\
11-15 years & 7 & 10.4 \\
\hline +16 years & 3 & 4.5 \\
\hline
\end{tabular}

\section{Institutions Served $^{1}$}

Private education and rehabilitation 40 center

\begin{tabular}{ll} 
Speech and language therapy clinics & 37 \\
Private hospital & 7 \\
Public hospital & 1 \\
University & 7 \\
Other & 3 \\
\hline
\end{tabular}

${ }^{1}$ Due to the fact that many SLTs served in more than one type of institution, the total number of frequencies in the category of institutions served was higher than $\mathrm{N}=67$. 


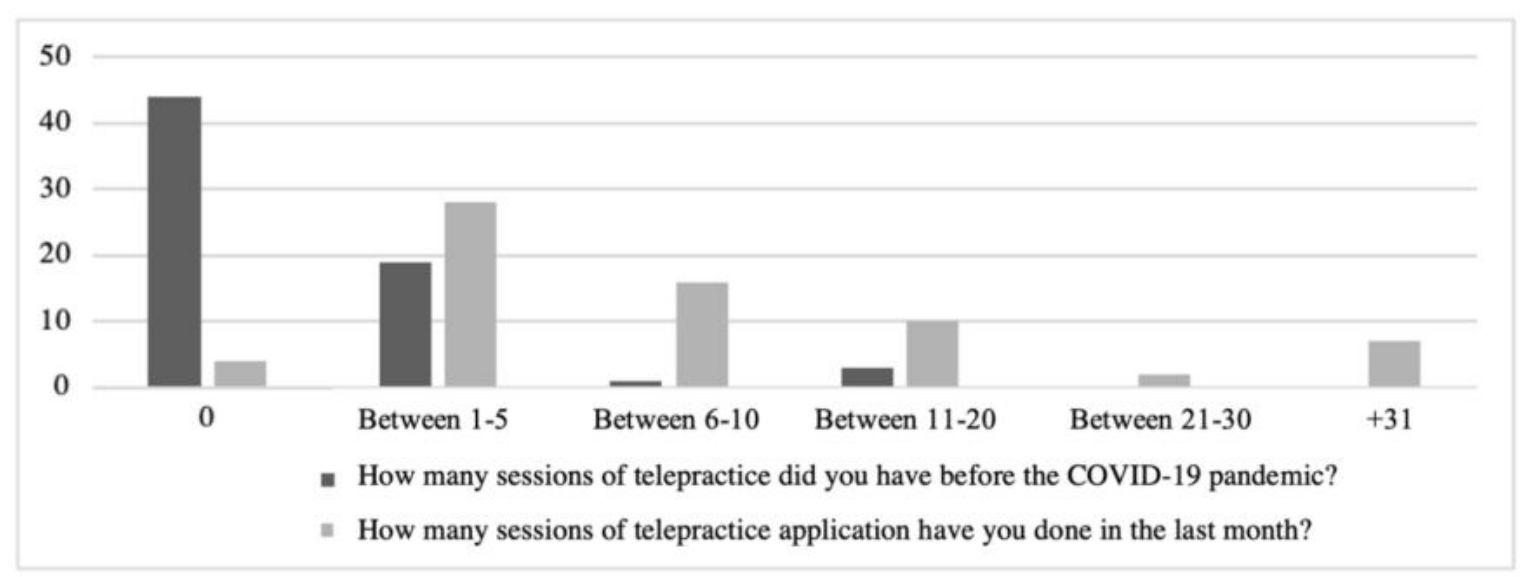

Fig. 1. Number of telepractice sessions before and after the COVID-19 pandemic.

ers at 'normal' $(\mathrm{n}=15)$ and 'very good' $(\mathrm{n}=14)$ level was almost equal.

How often did SLTs have online meetings before the COVID-19 pandemic?

According to the findings regarding the frequency of SLTs having online meetings before the COVID-19 pandemic, almost half of the participants reported that they had 'never' online meetings $(\mathrm{n}=33)$. 24 SLTs stated that they had 'almost never' online meetings and 10 SLTs stated that they had online meetings 'sometimes'.

How many telepractice sessions did SLTs have before and after the COVID-19 pandemic?

The comparative number of telepractice sessions performed by SLTs before and after the COVID-19 pandemic (in the last month) was given in Fig. 1. It was observed that almost half of the SLTs did not apply any telepractice sessions before the pandemic $(\mathrm{n}=44)$. The number of SLTs applying 1-5 sessions was only 19. After the pandemic, 28 SLTs performed between 1-5, 16 SLTs between 6-10 and 10 SLTs 1120 sessions. Four SLTs reported that they never performed telepractice after the pandemic.

What were the experiences and thoughts of SLTS about group telepractice?

According to the answers given by the participants to the questionnaire regarding group therapy applications, 65 SLTs stated that they had not used group therapy in telepractice applications before. SLTs preferred the most partially appropriate $(\mathrm{n}=20)$ and absolutely appropriate $(n=15)$ options regarding the application of group therapy in telepractice.
Which technological problems were encountered most frequently in telepractice?

According to the responses of the participants to the questionnaire regarding whether they had any problems during telepractice, it was observed that 46 SLTs experienced various problems during telepractice, and 21 SLTs did not have any problems. Considering the distribution of the problems faced by SLTs who reported having problems, internet disconnections $(n=31)$, sound problems $(n=30)$ and visual problems $(n=29)$ were experienced most commonly. The least problematic issues were reported as software-related problems $(\mathrm{n}=8)$, computer-related problems $(n=9)$ and other $(n=2)$ problems.

\section{Which teleconferencing softwares were used most fre- quently in telepractice?}

Considering the distribution of software preferred by the participants during telepractice applications, it was seen that the majority of them conducted their telepractice sessions via the Zoom $(n=53)$ application. The two most frequently used softwares following it were Whatsapp (Video Call) $(\mathrm{n}=30)$ and Skype $(\mathrm{n}=24)$. The frequency of the usage of FaceTime, Adobe Connect and other software was equal $(n=3)$. None of the participants reported that they used Webex and Discord programs.

How many minutes were the telepractice session duration determined?

Almost half of the SLTs $(n=35)$ reported that they generally determined the telepractice sessions as 30 minutes. The other SLTs stated that they had longer telepractice sessions of 40 minutes $(n=26), 45$ min- 


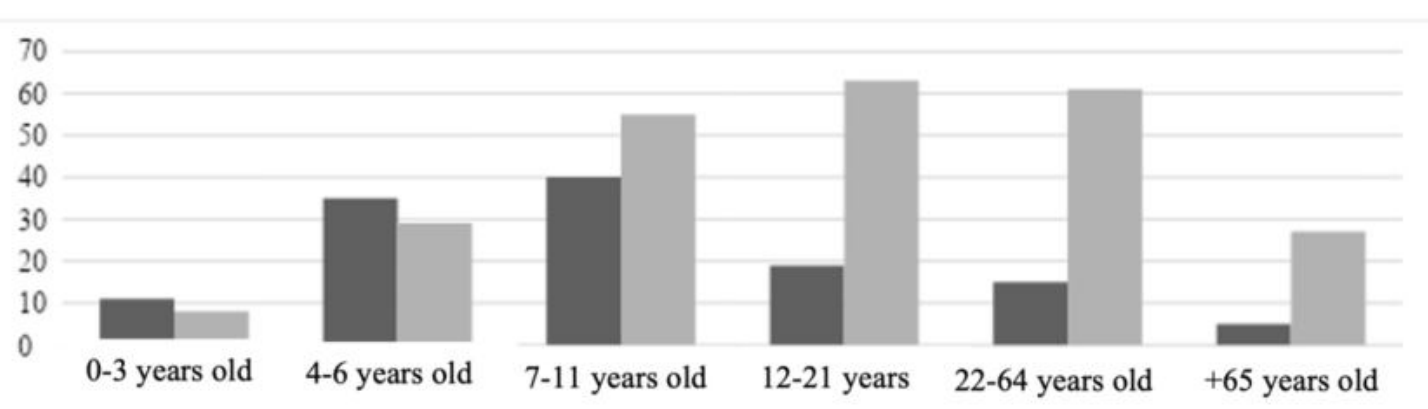

- To which age group/groups have you applied telepractice simultaneously?

- For which age groups do you think simultaneous telepractice is suitable?

Fig. 2. Age groups applied and appropriate for telepractice session.

utes $(n=22)$ and 60 minutes $(n=11)$. One participant marked the "other" option.

What were the age groups in which SLTS most frequently applied and deemed suitable for telepractice?

The age groups in which SLTs practiced telepractice and that they thought suitable for telepractice were given in Fig. 2 comparatively. The main age groups that SLTs found most suitable for telepractice were 12$21(n=63), 22-64(n=61)$ and 7-11 $(n=55)$. However, when looking at clinical applications, there was a situation that was not consistent with the age ranges found appropriate. Because, SLTs did the most telepractice sessions with children aged 7-11 $(n=40)$ and 4-6 $(\mathrm{n}=35)$, respectively.

In which client groups were helpful support needed?

The distribution of the client groups that the participants worked and needed assistant support during their telepractice applications was given in Figure 3. As seen in Fig. 3, 66 SLTs preferred to work with SSD in telepractice, 64 SLTs delayed speech and language (DSL) and 58 SLTs stuttering and other fluency disorders. Among these client groups studied, those that most require helpful support were SSD $(n=41)$, DSL $(\mathrm{n}=34)$, developmental communication disorders such as autism, etc.

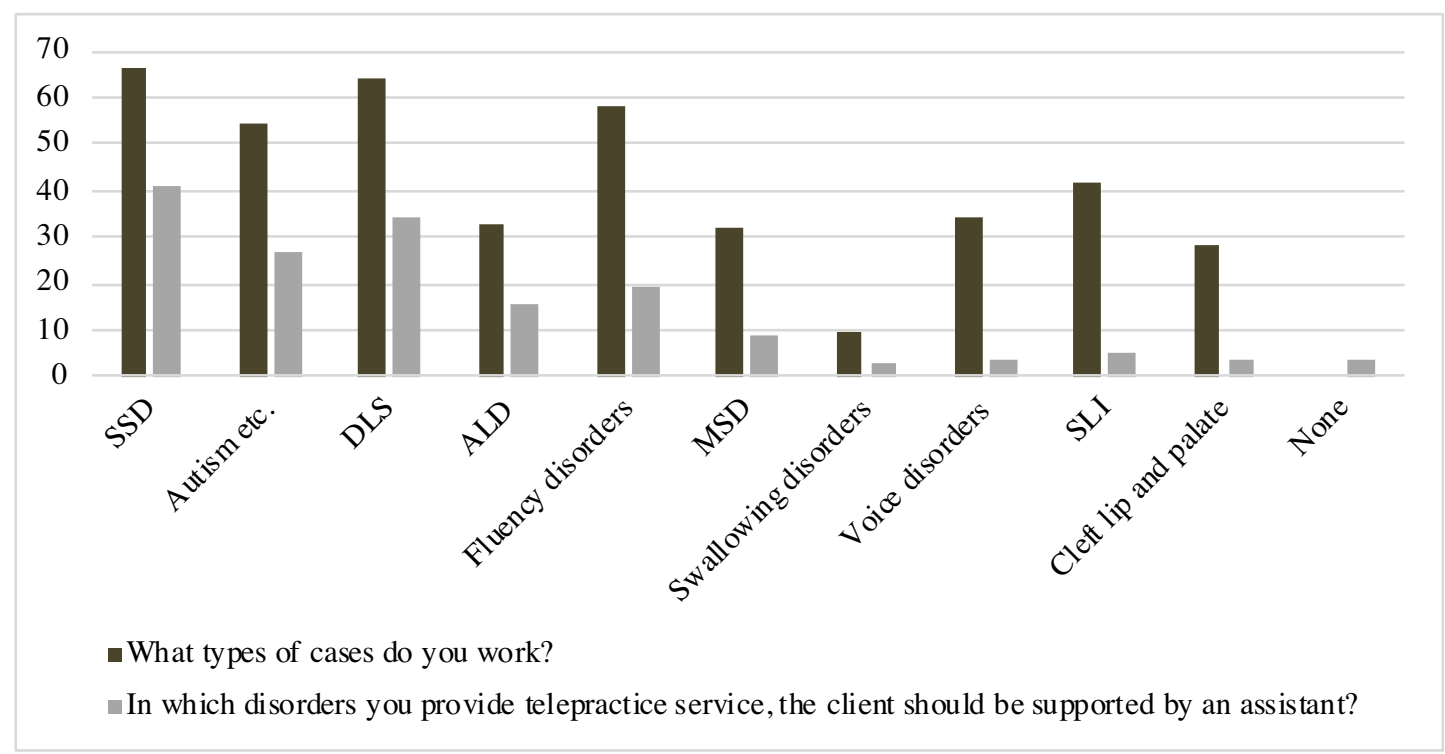

Fig. 3. Client groups that participants worked and required assistant support during telepractice. SSD $=$ Speech Sound Disorders, DSL = Delayed Speech and Language, ALD = Acquired Language Disorders, MSD = Motor Speech Disorders, SLI $=$ Specific Language Impairment, CLP $=$ Cleft Lip and Palate. 


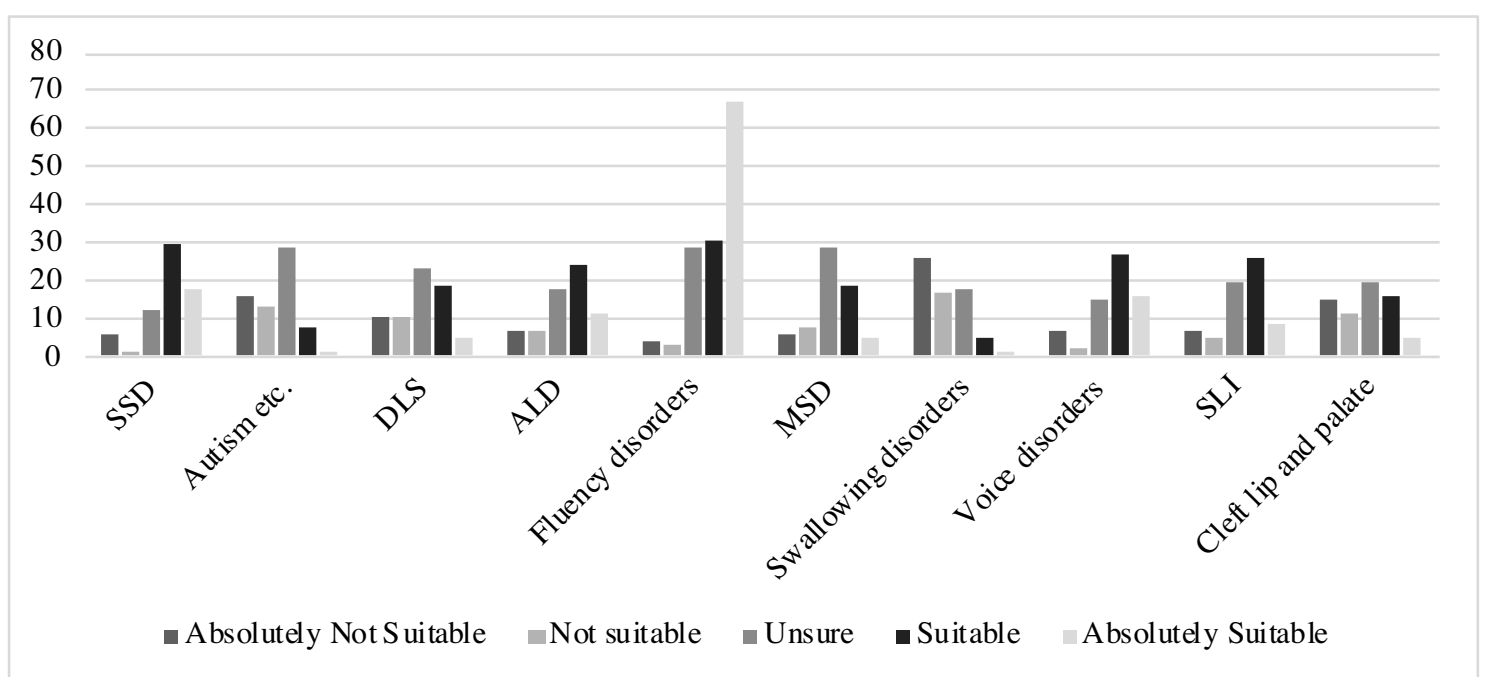

Fig. 4. Client groups that participants deem suitable for telepractice applications. SSD $=$ Speech Sound Disorders, DSL $=$ Delayed Speech and Language, ALD = Acquired Language Disorders, MSD = Motor Speech Disorders, SLI = Specific Language Impairment, CLP $=$ Cleft Lip and Palate.

\section{Which client groups was telepractice suitable for?}

As shown in Figure 4, the main disorders that SLTs most frequently marked the 'absolutely appropriate' options were fluency disorders $(n=31)$, voice disorders $(n=16)$ and $\operatorname{SSD}(n=18)$, respectively. The 'suitable' option was most frequently marked in speech and voice disorders $(\mathrm{n}=30)$, fluency disorders $(\mathrm{n}=$ $29)$, voice disorders $(n=27)$ and specific language impairment $(n=26)$. The disorder that was considered to be absolutely unsuitable for telepractice was swallowing disorder $(n=26)$.

\section{What were the satisfaction levels of SLTs in different}

subjects related to telepractice?

In the last part of the questionnaire, five questions were asked to evaluate the satisfaction of SLTs regarding telepractice. As can be seen in Fig. 5, the highest satisfaction level was related to the software used in telepractice $(n=26)$ and the lowest level of satisfaction was related to the financial gain provided by telepractice $(n=10)$. Most of the SLTs stated that they were partially satisfied with the level of using the clinician skills $(\mathrm{n}=24)$, the development of the clients $(n=25)$ and the financial gain $(n=24)$ in the telepractice process.

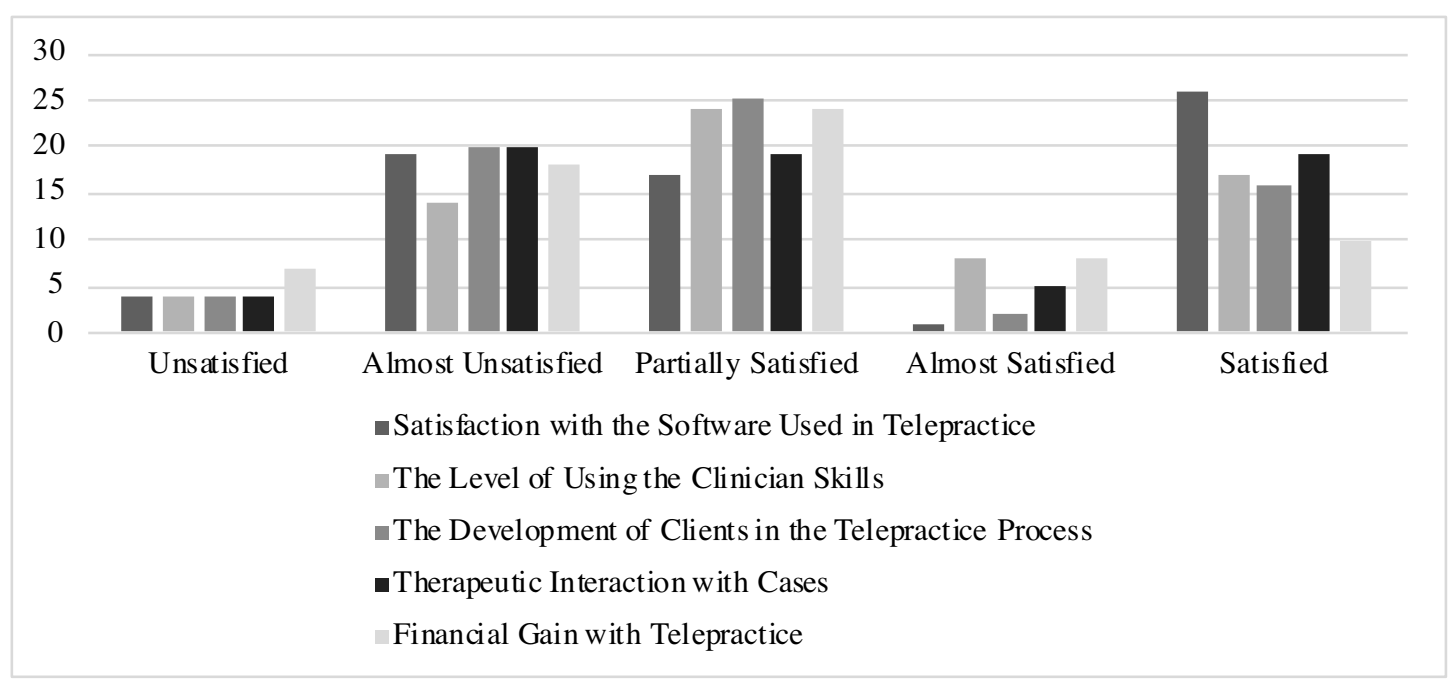

Fig. 5. Distribution of participants' satisfaction with telepractice according to different subjects 
Findings on the relationships between the telepractice sessions of SLTS after the COVID-19 pandemic and some variables

According to the first findings of the study, there was no relationship between 'gender' and 'perceived computer use skill' and 'the number of therapies they performed during the pandemic period' in SLTs (relatively; $\mathrm{x} 2=6.635, p=0.203 ; \mathrm{x} 2=13.592, p=0.734$ ).

There was a significant correlation between 'the frequency of online meetings conducted by SLTs before the pandemic' and 'the number of sessions they had during the pandemic period' ( $\mathrm{x} 2=19.028$; $\mathrm{p}=$ 0.012 ). There was a significant relationship between 'the telepractice sessions SLTs did before the pandemic' and 'the number of telepractice sessions they performed during the pandemic period' ( $\mathrm{x} 2=25.459$; $p=0.013$ ).

There is no significant relationship between 'the number of sessions' of SLTs in the last month and satisfaction from 'software used', 'development of clients', 'therapeutic communication' and 'financial gain' (relatively; $\mathrm{x} 2=18.991, p=0.640 ; \mathrm{x} 2=22.140, p=0.225$; $\mathrm{x} 2=21.509, p=0.216 ; \mathrm{x} 2=21.011, p=0.229)$. Only a significant relationship was found between "satisfaction with the level of using clinician skills" and "the number of sessions they had in the last month" of SLTs ( $\mathrm{x} 2=25.903 ; p=0.048)$.

\section{DISCUSSION}

In this study, the factors affecting the preferences and acceptance processes of language and speech therapists for telepractice in the COVID-19 pandemic were examined. As a result of the study, various findings were obtained regarding the demographic and professional information of the speech and language therapists, their general view on telepractice, the disorders they deem appropriate for telepractice, and their level of satisfaction with telepractice.

In this study, it was seen that the first two prominent findings were related to the familiarity and experience with telepractice. SLTs, who had pre-pandemic experience in the use of teleconferencing platforms socially or professionally, easily adapted to provide telepractice services under pandemic conditions. It was previously stated that experience with these tech- nologies has a determining role in attitudes towards future use $[4,30,32]$. The effect of initial experiences on attitudes and preferences was also reported in studies in other health areas [33-36]. At this point, in the light of the studies on the subject, the importance of SLTs following the model telepractice sessions and of encouraging them for first trials emerged [34].

Another important finding of the study was that there was a significant relationship between "satisfaction of using clinician skills" and "the number of sessions they did in the last month" of SLTs in an online environment. It was stated by some authors that telepractice might have a negative effect on clinician skills $[30,24,37]$. Although there was no relationship between "satisfaction with the development of the clients" and "the number of sessions they held in the last month", this finding could be considered within the scope of the Technology Acceptance Model [27] More online sessions of SLTs, who reported that they had been satisfied with using their clinical skills in telepractice, emphasized how important perceived usefulness and utility were on attitudes. This finding was consistent with the findings of some telepractice (telepractice, telerehabilitation) studies [4, 38-41]. It became prominent once again that the more clinicians take a technology useful, the easier it is to adapt to these technologies. This finding provided clinical implications for what kind of incentive studies could be done for SLTs to easily switch to telepractice services. It can provide evidence, in-service trainings, models and supervision for the effectiveness of the method in developing the first attitudes of SLTs towards telepractice.

Considering the platforms preferred by the participants to use during telepractice applications, it was seen that they mostly conducted telepractice sessions via the Zoom application. Then, the two most frequently used softwares were Whatsapp (video call) and Skype. FaceTime, Adobe Connect and other software were found to be the last and equally preferred in terms of usage frequency. In spite of this situation, in a qualitative study in the literature, thoughts about the usage and accessibility of Adobe Connect software were reported [42]. The fact that the standard version of Zoom is free to use in one-to-one calls and is easy to use compared to software such as Adobe Connect and Webex may have led the participants to this pref- 
erence. The issue that needs to be emphasized here is the importance of the "perceived convenience" factor within the scope of the Technology Acceptance Model [27]. The easier the usage of these systems is perceived in the beginning, the more positive user attitudes are [38] In their study, Bradford et al. [14] stated that most of the practitioners whose opinions they received initially doubted that telepractice would be effective and thought that the therapeutic relationship could not be established, but they concluded that their negative opinions on this issue changed in the opposite direction as the telepractice sessions progressed. Similarly, in our study, SLTs who performed telepractice sessions before the pandemic did not have any difficulty in providing telepractice services during the pandemic process.

SLTs reported that they most frequently used the standard version of Zoom, WhatsApp and Skype in telepractice. However, all these platforms do not have HIPAA (Health Insurance Portability and Accountability Act) certification. According to ASHA [5], the first issue that SLTs should look into when choosing these platforms is whether the systems have this security certificate. In this way, the security of the data regarding the client, the therapist or the service used will be ensured. Another issue considered secondary is that WhatsApp and Skype do not have file sharing and whiteboard features. This situation makes the applied telepractice more limited. Apart from the software used in providing telepractice service, technical issues are also important.

In this study, the most common technological problems that SLTs experienced during service delivery were internet disconnections, sound problems and video problems. These problems are highly anticipated problems in online usage in line with the literature [4, $43,24]$. Thus, the necessity of in-service training and how important it is to provide guidance and guides to SLTs revealed once again.

In this study, no relationship was found between the ages of SLTs and the frequency of telepractice sessions during the pandemic period. However, there are opinions that there is a relationship between these variables [18]. In Turkey, the SLT area is a developing area and an important part of personnel serving in this field is under 30 years of age. Thus, it was not possible to examine all participants by dividing them into certain age groups (eg, 20-40 and 40-60). It may be suggested future studies to examine the subject by considering different age groups.

SLTs reported the disorders in which telepractice can be used most effectively as speech voice disorders, fluency disorders and voice disorders, respectively. The least appropriate disorder was swallowing disorders. This situation is mostly compatible with the literature. In the field of research, it is seen that the disorders in which telepractice is effective are SSD $[44,45]$, fluency disorders [4, 15, 46, 47] and vocal disorders [13, 48-51] similar to our study. However, it is seen that there are many effectiveness studies in the literature, from autism [52-54] to swallowing disorder [55-57]. However, at this point, findings regarding user perspectives in terms of clients and clinicians in qualitative research are also extremely important. In this context, qualitative studies are also needed to determine the disorders for which telepractice is appropriate.

It was observed that the satisfaction levels of SLTs regarding the therapeutic relationship in telepractice were answered almost equally as "almost not satisfied", "partially satisfied" and "satisfied". This situation showed that the level of satisfaction of SLTs with the therapeutic relationship with the clients was not very high. In some studies in the literature, it was stated that the therapeutic relationship between the therapist and the client could be negatively affected in telepractice $[28,42,58]$. However, in telepractice, the therapeutic relationship between the client and the therapist needs to be studied in more detail. For example, although it was reported that telepractice might have some disadvantages in terms of therapeutic relevance [4], it is also important to examine which factors (e.g., clinician skills, natural approaches) minimize this disadvantage.

There was a significant relationship between the number of sessions that SLTs did in the last month and their level of using the skills of the clinician. With this finding, it was seen that the thoughts of SLTs on their level of using clinician skills were related to the number of sessions they had done. In this context, the increase in the telepractice experience of the therapist may lead to an increase in positive thoughts about the better use of the clinician's skills. In other words, it can be said that as the therapist's familiarity with the 
new service increases, he/she can use his/her skills regarding that service better over time.

With the increase in the number of sessions done by SLTs during the pandemic, their satisfaction level with the telepractice service they offer also increased. Especially in the high satisfaction level of the telepractice service they provide, e-helpers were of great importance. In this study, the most frequently needed disorder groups were SSD, DSL and autism etc. The younger age of the individuals in the relevant disorder groups may create a disadvantage in the processes of adapting and maintaining their cognitive or physical abilities to the service offered in front of the telepractice screen. This finding may be based on the assumption that the auxiliary support of SLTs in telepractice can facilitate efficient gain in the client. Moreover, it was found that SLTs had the most telepractice sessions with children aged 7-11 and 4-6, respectively. Considering that the disorder groups presenting in these age groups were disorders such as SSD, DSL and autism, it was seen that SLTs performed telepractice in the group where auxiliary support was most needed. However, the age groups that SLTs found most suitable for telepractice were 12-21, 22-64 and 7-11 years. An important reason why SLTs found these age groups suitable for telepractice may be that they do not need adult support in telepractice sessions applied for these age groups.

Finally, most of the participants stated that they did not do group therapy in telepractice applications before. Furthermore, half of the participants thought that telepractice was suitable for group therapy, while the other half stated that it was not. Group therapies differ in terms of therapy dynamics compared to individual therapies and contain different interaction elements. While individual therapies are based solely on client-therapist interaction, group therapies are based on the interaction of clients with one another and with the therapist. Since achieving these dynamics may require experience and competence for the therapist in telepractice processes compared to in-person therapy, SLTs may exhibit a timid attitude regarding the application of group therapy in telepractice.

\section{CONCLUSION AND RECOMMENDATIONS}

It was revealed that for SLTs, first trials on tele- conferencing platforms and familiarity with the method were decisive in future preferences for using telepractice. Using these systems for social purposes or to provide therapy services, SLTs were able to adapt to provide these services at an early stage even in a difficult period such as the COVID-19 pandemic. This finding supports the model [27] that TAM's "perceived easiness" will positively affect future attitudes. At this point, it may be persuasive that professional organizations such as DKTD (ASLT) [3] provide guidelines for clinicians, model demonstrations of practices and evidence of effectiveness. Implementation protocols, practical trainings and guidelines for dealing with potential problems can accelerate this adaptation process, taking into account factors such as the current conditions of the country's legal, cultural or SLT field.

According to the SLTs, telepractice is not suitable for many client groups. As research evidence showed, telepractice can be used as an effective means of serving a variety of client groups, from autism to swallowing disorders, when properly prepared $[22,59,60]$. At this point, factors such as multiple disabilities, mobility, cooperation, and the importance of e-helpers came to light in candidate clients for telepractice.

It was also observed that the teleconferencing platforms used by SLTs were not compatible with ASHA [6] guidelines. SLTs probably preferred the software they were most familiar with and the easiest to use. However, according to ASHA [6], the most critical issue in software selection is the use of HIPAA compliant softwares that ensures the security of health-related data. Beyond that, the lack of features such as screen sharing and whiteboards in these softwares used will reduce the quality of the service provided. The finding related to using clinician skills in telepractice can be evaluated in the context of TAM's perceived usefulness [27]. As SLTs believe that they use their skills effectively in telepractice, their attitudes towards the method will become more positive. Here again, clinicians should be provided with guidance and supervision on how to use the skills they use in the online environment as well, just like in in-person therapy.

\section{Authors' Contribution}

Study Conception: MEC; Study Design: MEC, İCY, AI; Supervision: MEC; Funding: MEC, İCY, AI; Materials: MEC, ICY; Data Collection and/or Processing: MEC, İCY; Statistical Analysis and/or Data Inter- 
pretation: MEC, İCY, AI; Literature Review: MEC, İCY, AI; Manuscript Preparation: MEC, İCY, AI and Critical Review: MEC, İCY, AI.

\section{Conflict of interest}

The authors disclosed no conflict of interest during the preparation or publication of this manuscript.

\section{Financing}

The authors disclosed that they did not receive any grant during conduction or writing of this study.

\section{Acknowledgement}

We thank Aylin Tamyaman (SLT) and İrem Bahar Koç (SLT) for actively being involved in the research by reaching appropriate participants and helping the collecting data process.

\section{REFERENCES}

1. World Health Organization. Naming the coronavirus disease (COVID-19) and the virus that causes it. 2020. Available at:https:/www.who.int/emergencies/diseases/novel-coronavirus2019/technical-guidance/naming-the-coronavirus-disease-(covid2019)-and-the-virus-that-causes-it. Accessed September 12, 2020.

2. TR. Ministry of Health, General Directorate of Public Health. COVID-19 Guide. COVID-19 (SARS-CoV-2 Infection) Guide. Scientific Committee Study. Available at: https:/covid19bilgi.saglik.gov.tr/depo/rehberler/COVID-19_Rehberi.pdf. Accessed September 13, 2020.

3. ASLT-Association of Speech and Language Therapists. COVID-19 pandemic guide for speech and language therapists. 2020 . Available

at: https:/www.dktd.org/tr/files/download/p1e6m6ogp1142oouinq9 175s12oh4.pdf. Accessed: September 15, 2020.

4. Cangi ME, Toğram B. Stuttering therapy through telepractice in Turkey: a mixed method study. J Fluency Disord 2020;66:105793.

5. ASHA-American Speech-Language-Hearing Association. Telepractice services and coronavirus/COVID-19. 2020. Available at: https://www.asha.org/practice/telepractice-services-andcoronavirus/. Accessed September 15, 2020.

6. ASHA-American Speech-Language-Hearing Association. Telepractice. 2020. Available at: https://www.asha.org/prpprinttemplate.aspx?folderid $=8589934956$. Accessed September 15, 2020.

7. Lin FC, Chien HY, Chen SH, Kao YC, Cheng PW, Wang CT. Voice therapy for benign voice disorders in the elderly: a randomized controlled trial comparing telepractice and conventional face-to-face therapy. J Speech Lang Hear Res 2020;63:2132-40. 8. Burns CL, Ward EC, Gray A, Baker L, Cowie B, Winter N, et al. Implementation of speech pathology telepractice services for clinical swallowing assessment: an evaluation of service outcomes, costs and consumer satisfaction. J Telemed Telecare 2019;25:545-51.

9. Bridgman K, Onslow M, O’Brian S, Jones M, Block S. Lidcombe program webcam treatment for early stuttering: a randomized controlled trial. J Speech Lang Hear Res 2016;59:932-9.

10. Finch E, Lethlean J, Rose T, Fleming J, Theodoros D, Cameron A, et al. Conversations between people with aphasia and speech pathology students via telepractice: A Phase II feasibility study. Int J Lang Commun Disord 2020;55:43-58.

11. Neely L, Rispoli M, Gerow S, Hong ER, Hagan-Burke S. Fidelity outcomes for autism-focused interventionists coached via telepractice: a systematic literature review. J Dev Phys Disabil 2017;29:849-74.

12. Pullins V, Grogan-Johnson S. A clinical decision making example: implementing intensive speech sound intervention for school-age students through telepractice. Perspect ASHA Spec Interest Groups 2017; 2:15-26.

13. Towey M. Speech therapy telepractice. In: Kumar S, Cohn E., eds. Telerehabilitation. Springer, London, 2013:pp.101-23.

14. Bradford NK, Caffery LJ, Taylor M, Meiklejohn J, Smith AC, Langbecker D. Speech-language pathology services delivered by telehealth in a rural educational setting: the school's perspective. J Int Soc Telemed eHealth 2018;6:e20.

15. Carey B, O’Brian S, Onslow M, Packman A, Menzies R. Webcam delivery of the Camperdown Program for adolescents who stutter: a phase I trial. Lang Speech Hear Serv Sch 2012;43:37080.

16. Mashima PA, Doarn CR. Overview of telehealth activities in speech-language pathology. Telemed J E Health 2008;14:110117.

17. Regina Molini-Avejonas D, Rondon-Melo S, de La Higuera Amato CA, Samelli AG. A systematic review of the use of telehealth in speech, language and hearing sciences. J Telemed Telecare 2015;21:367-76.

18. Tucker JK. Perspectives of speech-language pathologists on the use of telepractice in schools: the qualitative view. Int J Telerehabil 2012;4(2):47-60.

19. Lowe R, O'Brian S, Onslow M. Review of telehealth stuttering management. Folia Phoniatr Logop 2013;65:223-38.

20. Overby MS. Stakeholders' qualitative perspectives of effective telepractice pedagogy in speech-language pathology. Int $\mathrm{J}$ Lang Commun Disord 2018;53:101-12.

21. Wales D, Skinner, Hayman M. The efficacy of telehealth-delivered speech and language intervention for primary school-age children: a systematic review. Int J Telerehabil 2017;9:55-61.

22. Weidner K, Lowman J. Telepractice for adult speech-language pathology services: a systematic review. Perspect ASHA Spec Interest Groups 2020;5:326-38.

23. Houston KT. Telepractice in speech-language pathology. Plural Publishing, San Diego; 2013.

24. Tucker JK. Perspectives of speech-language pathologists on the use of telepractice in schools: quantitative survey results. Int J Telerehabil 2012;4:61-72.

25. Sun H, Zhang P. The role of moderating factors in user technology acceptance. Int J Hum Comput Stud 2006;64:53-78. 
26. Dunkley C, Pattie L, Wilson L, McAllister LA. Comparison of rural speech-language pathologists' and residents' access to and attitudes towards the use of technology for speech-language pathology service delivery. Int J Speech Lang Pathol 2010;12: 333-43.

27. Davis FD. Perceived usefulness, perceived ease of use, and user acceptance of information technology. MIS Quarterly 1989;13:319-40.

28. Freckmann A, Hines M, Lincoln M. Clinicians' perspectives of therapeutic alliance in face-to-face and telepractice speechlanguage pathology sessions. Int $J$ Speech Lang Pathol 2017; 19:287-96.

29. Akamoglu Y, Meadan H, Pearson JN, Cummings K. Getting connected: speech and language pathologists' perceptions of building rapport via telepractice. J Dev Phys Disabil 2018;30:569-85.

30. Irani F, Gabel R. Telerehabilitation: adult speech and swallowing disorders. In: Houston KT, eds. Telepractice in SpeechLanguage Pathology. Plural Publishing, San Diego; 2013: p.139. 31. Büyüköztürk Ş. Questionnaire development. J Turk Educ Sci 2005;3:133-51.

32. Alberta College of Speech-Language Pathologists and Audiologists (ACSLPA). Available at: www.acslpa.ca. Accessed May $15,2020$.

33. Bullock DR, Vehe RK, Zhang L, Correll CK. Telemedicine and other care models in pediatric rheumatology: an exploratory study of parents' perceptions of barriers to care and care preferences. Pediatric Rheumatol Online J 2017;15:55.

34. Campbell J, Theodoros D, Hartley N, Russell T, Gillespie N. Implementation factors are neglected in research investigating telehealth delivery of allied health services to rural children: a scoping review. J Telemed Telecare 2020;26:590-606.

35. Segrelles CG, López PD, Chiner E, Fernández FE, GrandaOrive JI. Acceptance of telemedicine among respiratory healthcare professionals. Eur Res Telemed 2017;6:147-55.

36. Taylor OD, Armfield NR, Dodrill P, Smith AC. A review of the efficacy and effectiveness of using telehealth for paediatric speech and language assessment. J Telemed Telecare 2014;20:405-12.

37. Valentine DT. Stuttering intervention in three service delivery models (direct, hybrid, and telepractice): two case studies. Int J Telerehabil 2014;6:51-63.

38. Almojaibel AA, Munk N, Goodfellow LT, Fisher TF, Miller KK, Comer AR, et al. Health care practitioners' determinants of telerehabilitation acceptance. Int J Telerehabil 2020;12:43-50.

39. Kowitlawakul Y. The technology acceptance model: predicting nurses' intention to use telemedicine technology (eICU). Comput Inform Nurs 2011;29:411-8.

40. Rho MJ, Choi I, Lee J. Predictive factors of telemedicine service acceptance and behavioral intention of physicians. Int $\mathrm{J}$ Med Inform 2014;83:559-71.

41. Zailani S, Gilani MS, Nikbin D, Iranmanesh M. Determinants of telemedicine acceptance in selected public hospitals in Malaysia: clinical perspective. J Med Syst 2014;38:111.

42. Hines M, Lincoln M, Ramsden R, Martinovich J, Fairweather C. Speech pathologists' perspectives on transitioning to telepractice: what factors promote acceptance? J Telemed Telecare
2015;21:469-73.

43. Jahromi ME, Ahmadian L. Evaluating satisfaction of patients with stutter regarding the tele-speech therapy method and infrastructure. Int J Med Inform 2018;115:128-33.

44. Crutchley S, Campbell M. Telespeech therapy pilot project: stakeholder satisfaction. Int J Telerehabil 2010;2:23-30.

45. Grogan-Johnson S, Schmidt AM, Schenker J, Alvares R, Rowan LE, Taylor J. A comparison of speech sound intervention delivered by telepractice and side-by-side service delivery models. Commun Disord Q 2013;34:210-20.

46. Carey B, O’BrianS, Onslow M, Block S, Jones M, Packman A. Randomized controlled non-inferiority trial of a telehealth treatment for chronic stuttering: the Camperdown Program. Int J Lang Commun Disord 2010;45:108-20.

47. Lewis C, Packman A, Onslow M, Simpson JM, Jones M. A phase ii trial of telehealth delivery of the lidcombe program of early stuttering intervention. Int J Med Inform Am J Speech Lang Pathol 2008; 17:139-49.

48. Halpern AE, Ramig LO, Matos CE, Petska-Cable JA, Spielman JL, Pogoda JM, et al. Innovative technology for the assisted delivery of intensive voice treatment (LSVT LOUD) for Parkinson disease. Am J Speech Lang Pathol 2012;21:354-67.

49. Mashima PA, Brown JE. Remote management of voice and swallowing disorders. Otolaryngol Clin North Am 2011;44:130516.

50. Theodoros DG, Constantinescu G, Russell TG, Ward EC, Wilson SJ, Wootton R. Treating the speech disorder in Parkinson's disease online. J Telemed Telecare 2006;12 (Suppl. 3):S388-91.

51. Tindall LR, Huebner RA, Stemple JC, Kleinert HL. Videophone-delivered voice therapy: a comparative analysis of outcomes to traditional delivery for adults with Parkinson's disease. Telemed J e-Health 2008;14:1070-7.

52. Higgins WJ, Luczynski KC, Carroll RA, Fisher WW, Mudford OC. Evaluation of a telehealth training package to remotely train staff to conduct a preference assessment. J Appl Behav Anal 2017;50:238-51.

53. Iacono T, Dissanayake C, Trembath D, Hudry K., Erickson $\mathrm{S}$, Spong J. Family and practitioner perspectives on telehealth for services to young children with autism. Stud Health Technol Inform 2016;231:63-73.

54. Parmanto B, Lewis AN Jr, Graham KM, Bertolet MH. Development of the telehealth usability questionnaire (TUQ). Int $\mathrm{J}$ Telerehabil 2016;8:3-10.

55. Cassel SG, Edd AJH. A pedagogical note: use of telepractice to link student clinicians to diverse populations. Int J Telerehabil 2016;8:41-8.

56. Malandraki GA, McCullough G, He X, McWeeny E, Perlman AL. Teledynamic evaluation of oropharyngeal swallowing. J Speech Lang Hear Res 2011;54:1497-505.

57. Perlman AL, Witthawaskul W. Real-time remote telefluoroscopic assessment of patients with dysphagia. Dysphagia 2002;17:162-7.

58. Hill AJ, Miller LE. A survey of the clinical use of telehealth in speech-language pathology across Australia. J Clin Pract Speech Lang Pathol 2012;14:110-7.

59. Burns CL, Ward EC, Hill AJ, Kularatna S, Byrnes J, Kenny 
LM. Randomized controlled trial of a multisite speech pathology telepractice service providing swallowing and communication intervention to patients with head and neck cancer: Evaluation of service outcomes. Head Neck 2017;39:932-9.
60. Ferguson J, Craig EA, Dounavi K. Telehealth as a model for providing behaviour analytic interventions to individuals with autism spectrum disorder: A systematic review. J Autism Dev Disord 2019;49:582-616. 